\title{
The Effect of Virtual Reality and Tetra- Ataxiometric Posturography Programs on Stroke Patients With Impaired Standing Balance
}

\author{
Yoon Bum Song, $\mathrm{MD}^{1}$, Min Ho Chun, MD, $\mathrm{PhD}^{1}$, Won Kim, $\mathrm{MD}^{1}$, \\ Sook Joung Lee, $\mathrm{MD}^{2}$, Jin Hwa Yi, $\mathrm{MD}^{1}$, Dae Hwan Park, $\mathrm{MD}^{1}$ \\ ${ }^{1}$ Department of Rehabilitation Medicine, Asan Medical Center, Seoul; \\ ${ }^{2}$ Department of Rehabilitation Medicine, Dong-A University Hospital, Busan, Korea
}

\begin{abstract}
Objective To investigate the effect of virtual reality (VR) and a tetra-ataxiometric posturography (Tetrax) program on stroke patients with impaired standing balance.

Methods Thirty acute stroke patients with impaired standing balance were recruited and randomly assigned to a VR, Tetrax, or control group. All patients received conventional balance training as a baseline; and VR and Tetrax patients received VR or Tetrax treatment, in addition. The primary outcome measures to evaluate the overall standing balance were the Berg Balance Scale (BBS) and the falling index (FI). The secondary outcome measures were the stability index (SI) and the weight distribution index (WDI), which were used to evaluate the balance status according to specific body positions. The FI, SI, and WDI were measured using the Tetrax instrument.

Results The BBS and FI scores were improved in all groups, with no significant differences between groups. In open-eyed positions, the VR group showed significantly greater improvement in SI and WDI scores than the control group $(\mathrm{p}<0.017)$. In closed-eyed positions, the Tetrax group showed significantly greater improvement in SI and WDI scores than the control group $(\mathrm{p}<0.017)$.

Conclusion The inclusion of VR and Tetrax programs did not lead to an overall benefit in balance. VR and Tetrax did, however, demonstrate a benefit in specific positions. A Tetrax program may benefit patients with abnormal proprioceptive function, whereas a VR program may benefit patients with normal sensory function.
\end{abstract}

Keywords Stroke, Postural balance, Virtual reality exposure therapy, Feedback

Received September 30, 2013; Accepted October 14, 2013

Corresponding author: Min Ho Chun

Department of Rehabilitation Medicine, Asan Medical Center, 88 Olympic-ro 43-gil, Songpa-gu, Seoul 138-736, Korea

Tel: +82-2-3010-3796, Fax: +82-2-3010-6964, E-mail: mhchun@amc. seoul.kr

(c) This is an open-access article distributed under the terms of the Creative Commons Attribution Non-Commercial License (http://creativecommons. org/licenses/by-nc/3.0) which permits unrestricted noncommercial use, distribution, and reproduction in any medium, provided the original work is properly cited.

Copyright $\odot 2014$ by Korean Academy of Rehabilitation Medicine

\section{INTRODUCTION}

Balance disorders after strokes are a major clinical concern. The risk of fall for hemiparesis patients is dramatically higher than for the general population during acute post-stroke hospitalization, during the rehabilitation period and later during community living [1]. There are specific risk factors for falls after a stroke: mental disorders, urinary incontinence, motor impairment, visuospa- 
tial neglect and postural stability [2]. Initial balance disability is also a strong predictor of function and recovery following a stroke [3].

Balance in stroke patients is compromised for several reasons, including the deterioration of motor and sensory functions on the affected side. As falling is a major post-stroke complication, balance training in stroke patients is essential [4]. There are various methods for balance training, including conventional balance training by physical therapy, training by tetra-ataxiometric posturography (Tetrax) and virtual reality (VR) training programs.

Neuro-developmental balance training, known as the 'Bobath approach' to balance training, is effective in improving weight distribution after stroke for up to 12 weeks [5].

VR technology is becoming increasingly popular for physical rehabilitation and motor control research. VR provides the opportunity for improvement in: ecological validity, stimulus control and consistency; real-time performance feedback; independent practice; stimulus and response modifications that are contingent on a user's physical abilities; and a safe testing and training environment. It also provides an opportunity for graduated exposure to stimuli, the ability to distract or augment the patient's attention and, importantly, patient motivation. It is known that visual inputs play an important role in maintaining balance. A number of studies have looked at the effect of VR on postural stability, but mainly in situations of visual-vestibular conflict rather than during normal VR [6].

Tetrax is based on the measurement and computerized elaboration of electronic signals emitted by four footplates, one for each heel and toe [7]. A Tetrax training program is known to improve standing stability. Increased postural sway is apparent when subjects are asked to close their eyes as compared to standing with eyes open. Likewise, sway-referencing visual information to head position leads to increased postural sway compared to normal visual inputs [8]. The Tetrax is also known as an effective balance-status measurement instrument. Thus, it is important to evaluate the effect of balance training using VR and Tetrax programs, in addition to conventional balance training.

\section{MATERIALS AND METHODS}

\section{Subjects}

Thirty stroke patients were recruited and randomly assigned to a VR, Tetrax or control group (Table 1). Inclusion criteria were hemiparesis resulting from a single cerebrovascular accident within 30 days that was not due to a trauma, brain tumor, surgery or any other etiology. Exclusion criteria were as follows: abnormal neuroophthalmologic findings and visual acuity problems after correction; a Berg Balance Scale (BBS) score below 40; an unstable medical condition; a history of musculoskeletal pathology affecting the lower extremities; poor cognitive function (Korean version of Mini-Mental State Examination <24); and hemispatial neglect.

\section{Study groups and training protocols}

All patients underwent conventional balance training five times per week, at 25 minutes per session. The VR group received additional VR treatment, three times per week, at 25 minutes per session, for three weeks. The Tetrax group received additional Tetrax treatment, three times a week, at 25 minutes per session, for three weeks.

In each VR session, the IREX (Vivid group, Toronto, Canada) was used. The VR program was composed of a television (TV) screen, camera and green screen as a background, along with red gloves. When the patient stood in front of the camera, the VR system reflected the patient on the TV screen. The patient was then instructed to perform a task. The red gloves or the patients themselves were recognized as markers for the tasks, allowing the patient to move their arms to accomplish the tasks. The VR program was composed of five tasks, which required the patient to move in such a way that their center of pressure (COP) was directed outside the feet. If the

Table 1. The demographic characteristics of patients

\begin{tabular}{lccc}
\hline & $\begin{array}{c}\text { Virtual } \\
\text { reality } \\
(\mathbf{n = 1 0})\end{array}$ & $\begin{array}{c}\text { Tetrax } \\
(\mathbf{n}=\mathbf{1 0})\end{array}$ & $\begin{array}{c}\text { Control } \\
(\mathbf{n}=\mathbf{1 0})\end{array}$ \\
\hline Sex (male:female) & $4: 6$ & $5: 5$ & $7: 3$ \\
\hline Age (yr) & $65.6 \pm 13.5$ & $60.6 \pm 18.2$ & $61.2 \pm 13.8$ \\
Time since stroke (day) & $12.7 \pm 3.2$ & $12.8 \pm 3.4$ & $13.2 \pm 3.4$ \\
\hline Stroke side (right:left)* & $1: 9$ & $0: 10$ & $1: 9$ \\
\hline
\end{tabular}

Values are presented as mean \pm standard deviation. ${ }^{*} \mathrm{p}<0.05$ by Fisher exact test. 
patient had weakness in the upper extremity, the tasks were adjusted, but the patient was still required to move so that their COP was directed outside of their feet. Each task was conducted for 5 minutes, with a 1-minute interval between tasks. One complete session took 30 minutes (Fig. 1).

In a Tetrax session, the patient was instructed to use the Tetrax (Sunlight Medical Ltd., Ramat Gan, Israel) instrument and its associated program. The patient was instructed to stand in the middle of four force plates, which were placed at the right front, right rear, left front, and left rear of the patient's feet. The patient was able to see the COP on the screen and was instructed to move it to accomplish the task. Each task had a duration of 5 minutes, with a 1-minute interval provided between tasks. One complete session took 30 minutes (Fig. 2).

\section{Outcome measurements}

Outcome measurements were obtained before and after the three weeks of training. The primary outcome measures were the BBS and falling index (FI) scores. The secondary outcome measures were the stability index (SI) and weight distribution index (WDI) scores. FI, SI, and
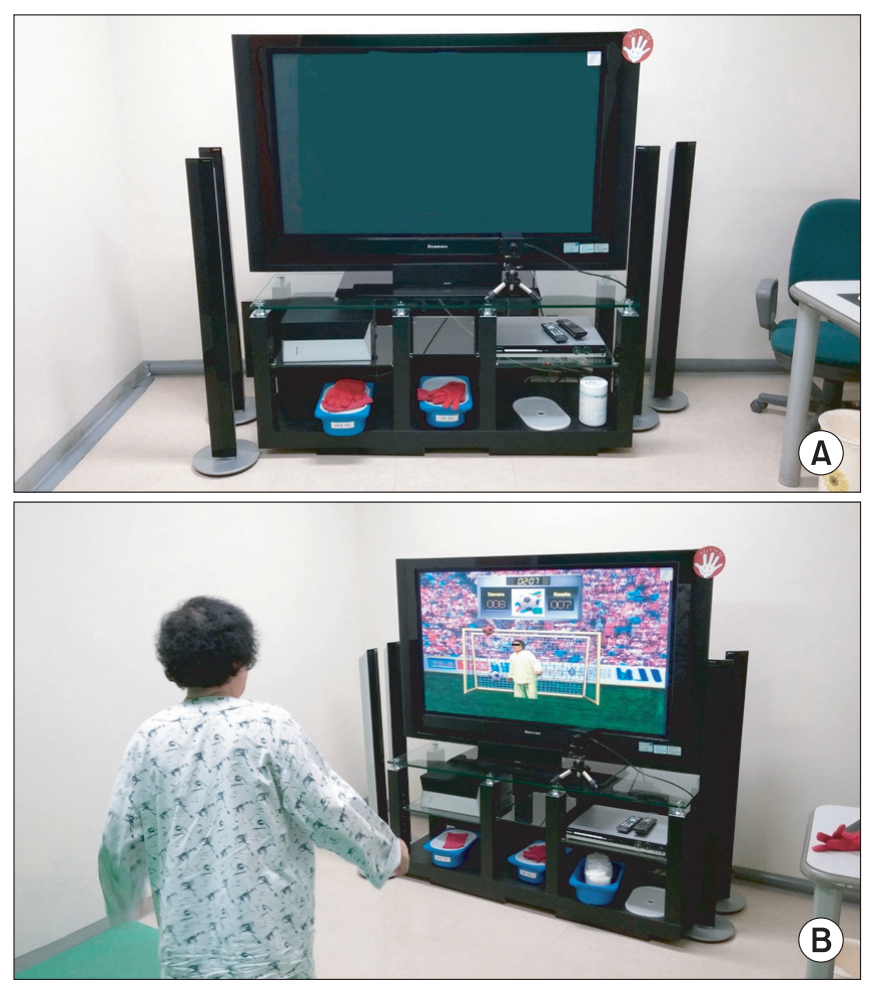

Fig. 1. (A) The IREX virtual reality instrument and (B) a patient using virtual reality program.
WDI scores were measured using the Tetrax instrument.

\section{Primary outcome}

The BBS measures the balance in three positions (sitting, standing, and changing position). It is composed of 14 categories, and the scores of each category are measured from 0 to 4 . The highest possible score is 56 , and the higher the score, the better the patient's balance.

The FI is a summation of the standard deviation of SI and the Fourier index (FIx) of lower medium and higher medium frequencies. FIx is obtained from a regression analysis of body sway using a Fourier transformation. The FIx value for lower medium frequency is high in vestibular dysfunction patients. The FIx value for higher medium frequency is high in patients with dysfunction of somatosensory response in the lower extremities and spine. Consequently, FI represents the quantitative risk of a fall, with a higher FI representing a greater likelihood of a fall.

\section{Secondary outcomes}

The SI measures the frequency of weight change in the four force plates and represents a patient's ability to adjust the position toward a more stable state. A higher score represents a more unstable body-balance control status.

The WDI measures the distribution of weight on the

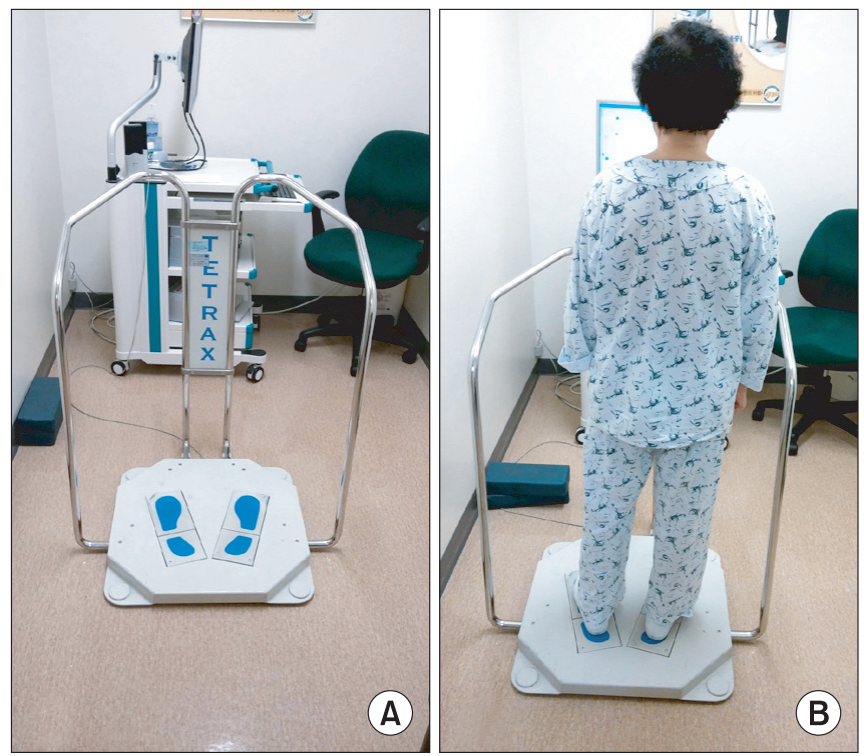

Fig. 2. (A) Tetra-ataxiometric posturography (Tetrax) instrument and (B) a patient using the Tetrax program. 
four force plates. The normal weight distribution is $25 \%$ on each plate. If a patient's weight distribution changes, it increases WDI and represents a more unstable bodybalance status.

\section{Positions used in Tetrax measurement}

The patients stood on the force plates with bare feet, arms by their side and with forward gaze. There were eight different configurations. The patients were asked to look forward with eyes open and closed (NO and NC). Next, they were asked to turn their head to the left and right (HL and HR) more than $45^{\circ}$. Next, they were asked to extend their head upward and downward (HF and HB). Finally, the patients were asked to stand on a foamrubber pillow with their eyes open and closed (PO and PC).

\section{Statistical analysis}

The data were analyzed with SPSS statistical software ver. 20.0 (SPSS Inc., Chicago, IL, USA). The demographic characteristics were analyzed by the chi-square test or the Fisher exact test. A p-value less than 0.05 was deemed to indicate statistical significance. The improvement of balance status from before to after the treatment was analyzed using Wilcoxon signed-rank test, and the differences between the three groups were analyzed using the Kruskal-Wallis test. A p-value less than 0.05 was deemed to indicate statistical significance for these tests. A posthoc analysis was also performed using the Mann-Whitney $U$ test to test individual differences between pairs of groups. For the post-hoc analysis, a p-value less than 0.017 was deemed to indicate statistical significance.

\section{RESULTS}

\section{General characteristics of patients}

The group characteristics were as follows: VR group: four males and six females, mean age of $65.6 \pm 13.5$ years,

Table 2. The Berg Balance Scale and falling index scores

\begin{tabular}{|c|c|c|c|c|c|c|}
\hline & \multicolumn{2}{|c|}{ Virtual reality } & \multicolumn{2}{|c|}{ Tetrax } & \multicolumn{2}{|c|}{ Control } \\
\hline & Pre & Post & Pre & Post & Pre & Post \\
\hline Berg Balance Scale* & $41.2 \pm 1.7$ & $48.3 \pm 3.5$ & $41.8 \pm 1.4$ & $49.4 \pm 2.3$ & $42.1 \pm 1.4$ & $47.9 \pm 2.3$ \\
\hline Falling index* & $82.9 \pm 7.2$ & $53.0 \pm 6.6$ & $84.0 \pm 8.9$ & $52.0 \pm 7.6$ & $81.8 \pm 8.1$ & $55.8 \pm 6.7$ \\
\hline
\end{tabular}

Values are presented as mean \pm standard deviation.

${ }^{*} \mathrm{p}<0.05$ in comparison between pre- and post-treatment by Wilcoxon signed-rank test.

Table 3. The stability index scores for various positions

\begin{tabular}{|c|c|c|c|c|c|c|c|}
\hline \multirow{2}{*}{ Position } & \multicolumn{2}{|c|}{ Virtual reality } & \multicolumn{2}{|c|}{ Tetrax } & \multicolumn{2}{|c|}{ Control } & \\
\hline & Pre & Post & Pre & Post & Pre & Post & \\
\hline NO & $56.2 \pm 23.7$ & $24.1 \pm 18.0$ & $52.3 \pm 27.0$ & $27.8 \pm 25.6$ & $49.6 \pm 12.3$ & $28.3 \pm 13.5$ & VR vs. Control* \\
\hline NC & $46.1 \pm 15.3$ & $26.5 \pm 12.4$ & $50.0 \pm 16.2$ & $28.1 \pm 24.9$ & $52.1 \pm 18.7$ & $30.8 \pm 13.9$ & \\
\hline $\mathrm{PO}$ & $56.9 \pm 14.3$ & $23.9 \pm 13.8$ & $56.2 \pm 20.9$ & $33.5 \pm 22.8$ & $51.7 \pm 17.9$ & $30.3 \pm 21.5$ & VR vs. Control* \\
\hline $\mathrm{PC}$ & $53.7 \pm 13.2$ & $33.9 \pm 15.5$ & $50.7 \pm 21.9$ & $22.6 \pm 23.9$ & $57.7 \pm 20.4$ & $41.2 \pm 21.0$ & Tetrax vs. Control \\
\hline HR & $57.7 \pm 18.5$ & $39.1 \pm 11.1$ & $51.7 \pm 16.3$ & $34.1 \pm 14.8$ & $57.8 \pm 16.6$ & $39.9 \pm 10.0$ & \\
\hline HL & $55.1 \pm 10.4$ & $33.6 \pm 15.7$ & $57.1 \pm 23.4$ & $34.8 \pm 25.8$ & $57.7 \pm 22.5$ & $35.9 \pm 27.3$ & \\
\hline HB & $53.1 \pm 15.8$ & $35.8 \pm 22.8$ & $51.2 \pm 11.7$ & $34.4 \pm 15.0$ & $52.6 \pm 10.1$ & $26.2 \pm 12.4$ & \\
\hline $\mathrm{HF}$ & $58.6 \pm 18.2$ & $29.9 \pm 12.3$ & $53.4 \pm 10.1$ & $25.8 \pm 16.8$ & $56.1 \pm 11.7$ & $28.4 \pm 12.8$ & \\
\hline
\end{tabular}

Values are presented as mean \pm standard deviation.

VR, virtual reality; NO, standing with eyes open; NC, standing with eyed closed; PO, standing on pillow with eyes open; PC, standing on pillow with eyes closed; HR, standing with eyes closed with head rotation to the right side; HL, standing with eyes closed with head rotation to the left side; HB, standing with eyes closed with neck extension; HF, standing with eyes closed with neck flexion.

${ }^{*} \mathrm{p}<0.017$ by Mann-Whitney U test (for example, VR vs. control means the difference between VR and control group is statistically significant at $\mathrm{p}=0.017$ in post-hoc analysis). 
Table 4. The weight distribution index scores for various positions

\begin{tabular}{|c|c|c|c|c|c|c|c|}
\hline \multirow{2}{*}{ Position } & \multicolumn{2}{|c|}{ VR } & \multicolumn{2}{|c|}{ Tetrax } & \multicolumn{2}{|c|}{ Control } & \\
\hline & Pre & Post & Pre & Post & Pre & Post & \\
\hline NO & $12.7 \pm 1.4$ & $8.2 \pm 1.4$ & $11.7 \pm 3.6$ & $9.7 \pm 2.7$ & $11.3 \pm 2.2$ & $9.6 \pm 2.2$ & VR vs. Control* \\
\hline NC & $12.3 \pm 1.3$ & $9.8 \pm 2.2$ & $12.6 \pm 2.1$ & $7.9 \pm 1.7$ & $11.7 \pm 2.1$ & $9.9 \pm 1.2$ & Tetrax vs. Control* \\
\hline PO & $12.5 \pm 1.2$ & $7.7 \pm 1.4$ & $11.7 \pm 2.4$ & $9.3 \pm 2.0$ & $11.6 \pm 3.0$ & $9.5 \pm 3.3$ & VR vs. Control* \\
\hline PC & $11.5 \pm 1.2$ & $9.7 \pm 2.5$ & $12.1 \pm 2.4$ & $8.2 \pm 2.7$ & $11.2 \pm 3.7$ & $9.5 \pm 2.9$ & Tetrax vs. Control* \\
\hline HR & $12.2 \pm 1.4$ & $9.8 \pm 1.3$ & $12.5 \pm 2.5$ & $10.8 \pm 1.4$ & $10.8 \pm 1.6$ & $9.7 \pm 0.8$ & \\
\hline HL & $12.5 \pm 1.3$ & $9.7 \pm 1.5$ & $11.7 \pm 3.2$ & $9.1 \pm 2.5$ & $11.4 \pm 2.1$ & $9.1 \pm 2.0$ & \\
\hline HB & $10.1 \pm 2.5$ & $9.1 \pm 2.3$ & $11.1 \pm 2.3$ & $9.8 \pm 2.1$ & $11.1 \pm 2.0$ & $10.1 \pm 2.7$ & \\
\hline $\mathrm{HF}$ & $11.2 \pm 1.9$ & $9.6 \pm 1.3$ & $12.7 \pm 3.5$ & $11.0 \pm 2.2$ & $10.3 \pm 2.6$ & $9.0 \pm 1.2$ & \\
\hline
\end{tabular}

Values are presented as mean \pm standard deviation.

VR, virtual reality; NO, standing with eyes open; NC, standing with eyed closed; PO, standing on pillow with eyes open; PC, standing on pillow with eyes closed; HR, standing with eyes closed with head rotation to the right side; HL, standing with eyes closed with head rotation to the left side; HB, standing with eyes closed with neck extension; HF, standing with eyes closed with neck flexion.

${ }^{*} \mathrm{p}<0.017$ by Mann-Whitney U test (for example, VR vs. control means the difference between VR and control group is statistically significant at $\mathrm{p}=0.017$ in post-hoc analysis).

time since stroke of $12.7 \pm 3.2$ days, one right-side stroke and nine left-side strokes; Tetrax group: five males and five females, mean age of $60.6 \pm 18.2$ years, time since stroke of 12.8 \pm 3.4 days, all strokes on the left side; and the control group: seven males and three females, mean age of $61.2 \pm 13.8$ years, time since stroke of $13.2 \pm 3.4$ days, one right-side stroke and nine left-side strokes.

There were no significant inter-group differences in sex, age, and time since stroke. However, there was a significantly higher number of strokes on the left side (Table 1).

\section{Training effects}

There was a significant improvement in BBS and FI after treatment in all three groups $(\mathrm{p}<0.05)$, but there was no significant difference between the three groups (Table 2).

In open-eyed positions, the VR group showed significantly greater improvement in SI and WDI scores than the control group ( $\mathrm{p}<0.017)$, but with no difference between the Tetrax and control groups. In closed-eyed positions, the Tetrax group showed significantly greater improvement in SI and WDI scores than the control group $(\mathrm{p}<0.017)$, but there was no difference between the VR and control groups.

The change in head position (HR, HL, HB, and HF) did not show a difference in SI and WDI scores between the three groups (Tables 3, 4).

\section{DISCUSSION}

The VR, Tetrax, and conventional balance-training programs are known to be effective in improving balance. This is the first study investigating the effect of adding VR and Tetrax to conventional training.

We found that balance was improved in all three balance-training modalities. However, the addition of a VR and Tetrax program to conventional training did not show additional benefit to the patient's balance status. In the study of Yang et al. [9], COP-related measures and the symmetric index during quiet stance were considered, either on the VR treadmill or on traditional treadmill training. They found that there was no difference in improving the COP displacement between the two groups. They considered that these results arose from the initial stability of the patients. All the patients in their study could ambulate independently without the use of assistive devices. In our study also, patients were excluded if with BBS score of less than 40 , but patients were not excluded if they used an assistive device. However, all patients had a relatively high balance skill, and the same phenomenon as in the study of Yang et al. [9] may be present in this study. We also found that there was no improvement in SI and WDI in the positions that required head movements (HR, HL, HB, and HF). Because the BBS and FI represent the overall balance status, the summation of parameters 
in these positions could dilute the training effects.

However, two modalities had distinct effect with the patients in specific positions. On the VR program, the SI and WDI scores showed relatively greater improvement in open-eyed positions. On the Tetrax program, the SI and WDI scores showed relatively greater improvement in closed-eye positions. In this study, the balance training was designed to move the COP voluntarily in VR and Tetrax tasks. Winstein et al. [10] showed that balance skill training using static tasks did not lead to improvement in balance skill for performing dynamic tasks. Yang et al. [9] also suggested that the dynamic training would be necessary to improve dynamic balance control, but not static balance control. A plausible explanation for these observations is that balance skill has separate neural pathways for task-specific postural control learning and for static and dynamic balance control.

In the VR program, the patients could not see their COP on the screen, but they were able to move the COP without limitation. The tasks were designed to create more movement than that of the Tetrax program. The movements of patient's body and feet were not limited to within the instrument, and they could move their body and feet freely on the floor. Hence, the beneficial effects of VR training are thought to be due to stimulating the dynamic balance control. Consequently, the SI and WDI scores were improved in open-eyed positions.

Conversely, in the Tetrax program, the patient stands on four fixed flat force plates. The COP can be seen by the patient on the screen, and patients can accomplish the required tasks by moving the COP. Patients can move their COP voluntarily, but within the limited range of the fixed Tetrax instrument. Their feet are to be placed on the force plate to accomplish the task. Hence, the beneficial effects of Tetrax training are thought to be due to stimulating the static balance control. Consequently, the SI and WDI scores were improved in closed-eyed positions.

The results presented in this paper should be interpreted within the study limitations. First, the BBS and FI scores did not show statistically significant improvement. This may be due to a dilution effect of the balance status in other positions (HR, HL, HB, and HF). Since the sample size in this study was limited, the dilution effect could be higher than the effect of training. A larger sample size may resolve this issue. Second, the FI, SI, and WDI scores were measured using the Tetrax instrument. As the pa- tients in the Tetrax group used the same instrument in the training, this added instrument familiarity may have led to their having an advantage in terms of better control on the force plates. However, the SI and WDI scores showed significant improvement in NO and PO, so the effect of this familiarization may have been minimal. Third, the vast majority of patients had suffered a leftsided stroke. Since neglect could influence the balance status, we excluded patients with hemispatial neglect. Of the thirty patients, only two patients had right-sided strokes with no hemispatial neglect on evaluation. Because of the dominance of left-sided strokes, our results may have only limited applicability to patients with rightsided strokes.

In conclusions, the inclusion of VR and Tetrax programs did not lead to an overall benefit in balance. However, VR and Tetrax did demonstrate benefit in specific positions. The SI and WDI scores were improved in the VR program in open-eyed positions and in the Tetrax program in closed-eyed positions. A Tetrax program may benefit patients with abnormal proprioceptive function, whereas a VR program may benefit patients with normal sensory function.

\section{CONFLICT OF INTEREST}

No potential conflict of interest relevant to this article was reported.

\section{REFERENCES}

1. Tyson SF, Hanley M, Chillala J, Selley AB, Tallis RC. The relationship between balance, disability, and recovery after stroke: predictive validity of the Brunel Balance Assessment. Neurorehabil Neural Repair 2007;21:341-6.

2. Yelnik AP, Le Breton F, Colle FM, Bonan IV, Hugeron C, Egal V, et al. Rehabilitation of balance after stroke with multisensorial training: a single-blind randomized controlled study. Neurorehabil Neural Repair 2008;22:468-76.

3. Yost KJ, Cheville AL, Weaver AL, Al Hilli M, Dowdy SC. Development and validation of a self-report lowerextremity lymphedema screening questionnaire in women. Phys Ther 2013;93:694-703.

4. Weerdesteyn V, de Niet M, van Duijnhoven HJ, Geurts 
AC. Falls in individuals with stroke. J Rehabil Res Dev 2008;45:1195-213.

5. Mudie MH, Winzeler-Mercay U, Radwan S, Lee L. Training symmetry of weight distribution after stroke: a randomized controlled pilot study comparing taskrelated reach, Bobath and feedback training approaches. Clin Rehabil 2002;16:582-92.

6. Keshner EA. Virtual reality and physical rehabilitation: a new toy or a new research and rehabilitation tool? J Neuroeng Rehabil 2004;1:8.

7. Kohen-Raz R. Application of tetra-ataxiametric posturography in clinical and developmental diagnosis. Percept Mot Skills 1991;73:635-56.
8. Black FO, Wall C 3rd, Nashner LM. Effects of visual and support surface orientation references upon postural control in vestibular deficient subjects. Acta Otolaryngol 1983;95:199-201.

9. Yang S, Hwang WH, Tsai YC, Liu FK, Hsieh LF, Chern JS. Improving balance skills in patients who had stroke through virtual reality treadmill training. Am J Phys Med Rehabil 2011;90:969-78.

10. Winstein CJ, Gardner ER, McNeal DR, Barto PS, Nicholson DE. Standing balance training: effect on balance and locomotion in hemiparetic adults. Arch Phys Med Rehabil 1989;70:755-62. 